\title{
Sigmoid Sinus Thrombosis Followed by Splenic Infarction Due to Imatinib Therapy in a Patient with Gastrointestinal Stromal Tumor; Hematological Side Effects of Imatinib
}

\author{
Hatice Yılmaz ${ }^{1}$, Güzin Demirağ ${ }^{1}$, and Ali Yılmaz ${ }^{2}$ \\ ${ }^{1}$ Department of Medical Oncology, Ondokuz Mayıs University Faculty of Medicine, Samsun, Turkey; ${ }^{2}$ Department of \\ Medical Oncology, Atatürk University Faculty of Medicine, Erzurum, Turkey
}

Corresponding author: Dr. Hatice Y1lmaz, Orcid id: 0000-0001-5341-5646, Department of Medical Oncology, Ondokuz May1s
University Faculty of Medicine; Samsun, Turkey, 55270; TEL: (+90)5331511093; email: drhaticeyilmaz19@ gmail.com

Received, December 7, 2020; Revised, March 23, 2021; Accepted, March 24, 2021; Published, March 29, 2021

\begin{abstract}
Imatinib has an important place as an adjuvant therapy as well as in the treatment of metastatic disease caused by gastrointestinal stromal tumor (GIST), which is one of the common mesenchymal tumors of the gastrointestinal tract. Imatinib is a tyrosine kinase inhibitor and is generally well tolerated. However, it can cause some serious adverse effects. The most common of these are edema on the face and legs, headache, fatigue, nausea, vomiting, and rash on the skin. The most serious side effects, albeit less common, are gastrointestinal or intraabdominal bleeding. However, thrombotic events such as sigmoid sinus thrombosis and splenic infarction are extremely rare. The current report presents a patient with GIST who is treated with imatinib $400 \mathrm{mg} /$ day. The patient presented with edema on the face and headache in the second month of imatinib therapy, after which she was diagnosed with sigmoid sinus thrombosis. The patient who presented with abdominal pain approximately three months later developed splenic infarction. She was administered acetylsalicylic acid, supplemental oxygen (O2) in the first episode of thrombosis, and imatinib therapy was discontinued. The patient's complaints and thrombus regressed, after which imatinib therapy was resumed. She was administered intravenous hydration, supplemental oxygen, analgesics, and imatinib therapy was discontinued after the patient sustained splenic infarction. After resolution of sigmoid sinus thrombosis and the regression of splenic infarction area, the patient was switched to sunitinib therapy. She is attending routine control visits. Sigmoid sinus thrombosis and splenic infarction should be kept in mind as a rare cause of headache and abdominal pain in patients treated with imatinib, and detailed neurological and gastrointestinal evaluation should be performed.
\end{abstract}

\section{INTRODUCTION}

Gastrointestinal stromal tumor (GIST) originating from the interstitial cells of Cajal is a mesenchymal malignancy accounting for the majority of gastrointestinal system (GIS) soft tissue sarcomas [1]. GIST is usually caused by a mutation in protooncogenes KIT (CD117) and platelet-derived growth factor receptor alpha (PDGFR). It is commonly seen in the stomach, small intestine, rectum, and less commonly in other parts of the GIST [2]. Imatinib, which was originally approved for the treatment of metastatic disease and then as an adjuvant therapy, has an important place in the treatment of patients with GIST [1]. Imatinib is a selective protein kinase inhibitor, which inhibits BCR-ABL autophosphorylation and blocks the effects of BCR-ABL oncoprotein. Imatinib binds to amino acids in the ABL kinase region and blocks the binding of adenosine triphosphate, keeping the BCR-ABL protein in an inactive form. Thus, adenosine prevents phosphate transfer from triphosphate and blocks signal conduction pathways, causing cell growth, arrest, and cell death. It also blocks the transmembrane receptor KIT and PDGF [3]. Although imatinib is generally a well-tolerated drug, it can cause some serious side effects. Cardiovascular side effects (11-86\%) and edema in the face (especially in the periorbital region) and lower extremities are common side effects during imatinib therapy. Central nervous system (CNS) side effects include pain, fatigue, headache, and insomnia. GIS symptoms such as dyspepsia, nausea, vomiting, diarrhea, and skin symptoms such as rash, erythema are other side effects related to imatinib therapy [3]. The most common hematological side effects are bleeding (3-53\%), neutropenia (16-48\%), leukopenia (5$47 \%$ ), anemia (32-35\%), and thrombocytopenia (1$32 \%$ ) [4]. GIS and/or intra-abdominal hemorrhage, which is thought to occur as a result of damage to the vascular structure of the tumor due to the rapid effect of imatinib, is considered to be the most serious side effects. The tendency to bleeding in the 
natural course of advanced stage GISTs seems to be another reason. However, thrombotic events associated with imatinib therapy are extremely rare. The current report presents a case of sigmoid sinus thrombosis with concurrent splenic infarction associated with imatinib therapy, which has not been reported in the literature to the best of our knowledge.

\section{CASE PRESENTATION}

A 76-year-old female patient, who was on an adjuvant therapy with imatinib $400 \mathrm{mg} / \mathrm{day}$ after being diagnosed with a GIST localized in the stomach, presented with a one-week history of headache and facial edema that started two months into the therapy. The patient had a history of hypertension and was using an angiotensin receptor blocker. The patient did not have a history of smoking and/or alcohol use, and physical examination revealed normal findings other than periorbital edema. Biochemistry, complete blood count, and complete urine analysis revealed normal findings. Diffusion magnetic resonance imaging of the brain revealed slow flow and an increased signal intensity extending from the left transverse sinus to sigmoid sinus that was suggestive of a thrombus. Magnetic resonance venography, which was performed to confirm the diagnosis, did not detect blood flow in the beginning of the left transfer sinus and the left sigmoid sinus, and revealed a partial thrombus allowing blood flow in the left sigmoid sinus (Figure 1). The patient was therefore placed on a therapy with acetylsalicylic acid $100 \mathrm{mg}$ /day and supplemental $\mathrm{O} 2$ at a rate of 2-4 L/min, and imatinib was discontinued. The headache and facial edema disappeared two weeks after the initiation of the revised therapy, and imatinib therapy was resumed at a dose of 200 $\mathrm{mg}$ /day which was three weeks later titrated up to $400 \mathrm{mg} /$ day. The patient, who had no symptoms and physical examination findings, was discharged. She was called for monthly controls after being discharged. Physical examination, hemogram, and biochemical tests were performed at each visit. Routine follow-up of the patient was continued until when she was re-admitted with the complaints of facial edema and abdominal pain 3 months after resuming imatinib therapy. Physical examination revealed normal findings except for a left upper quadrant abdominal tenderness. Laboratory workup including complete blood count, electrolytes, lipid profile, thyroid function tests, liver and kidney function tests were within normal limits. The erect abdominal plain film and abdominal ultrasound revealed normal findings, and computed tomography (CT) scans of the abdomen revealed an area of ischemia-infarction in the superior-posterior region of the spleen measuring $35 \times 16 \mathrm{~mm}$ in size and an accessory splenic tissue inferior to the spleen measuring 19x14 mm in size (Figure 2). Imatinib therapy was therefore discontinued. Intravenous hydration, oxygen supplementation, and paracetamol were initiated. Hereditary thrombophilia tests (protein C, protein $\mathrm{S}$, antithrombin III, homocysteine, factor $\mathrm{V}$ Leiden mutation, prothrombin G20210A mutation) to investigate the etiologic cause were normal, and the patient tested negative for antinuclear antibodies, anti-neutrophil cytoplasmic antibodies, rheumatoid factor, anti-phospholipid antibodies, and hepatitis markers. In addition, CD55 and CD59 levels studied by flow cytometry were found to be normal. Echocardiography, electrocardiography, and peripheral blood smear revealed normal findings. Control abdominal CT scans obtained after the treatment revealed shrinkage of the infarct area in the spleen, and control MR venography of the brain showed blood flow in the left sigmoid sinus. The patient did not have complaints during the follow-up period., and at this stage, she was put on sunitib instead of imatinib. The patient reported no complaints or a new thrombotic event in subsequent control visits. The follow-up and treatment of the patient is continued.

\section{DISCUSSION}

Imatinib, which has become the standard of care in patients diagnosed with GIST and chronic myeloid leukemia, is a tyrosine kinase inhibitor that is better tolerated than the cytotoxic agents. The adverse effects associated with imatinib therapy are usually reversible and do not necessitate drug discontinuation or dose reduction. On the other hand, gastrointestinal or intra-abdominal hemorrhages associated with imatinib therapy which often occur as a result of tumor necrosis are rarer but fatal complications $[5,6]$. In a study of 95 patients with GIST receiving imatinib therapy, Schlemmer et al. showed that patients with gastrointestinal side effects constituted the majority of patients experiencing side effects, and gastrointestinal side effects were followed by general disorders, musculoskeletal and connective tissue disorders, and skin and subcutaneous tissue disorders [7]. However, they showed CNS disorders such as dizziness, headache, and paraesthesia associated with imatinib therapy in 14 


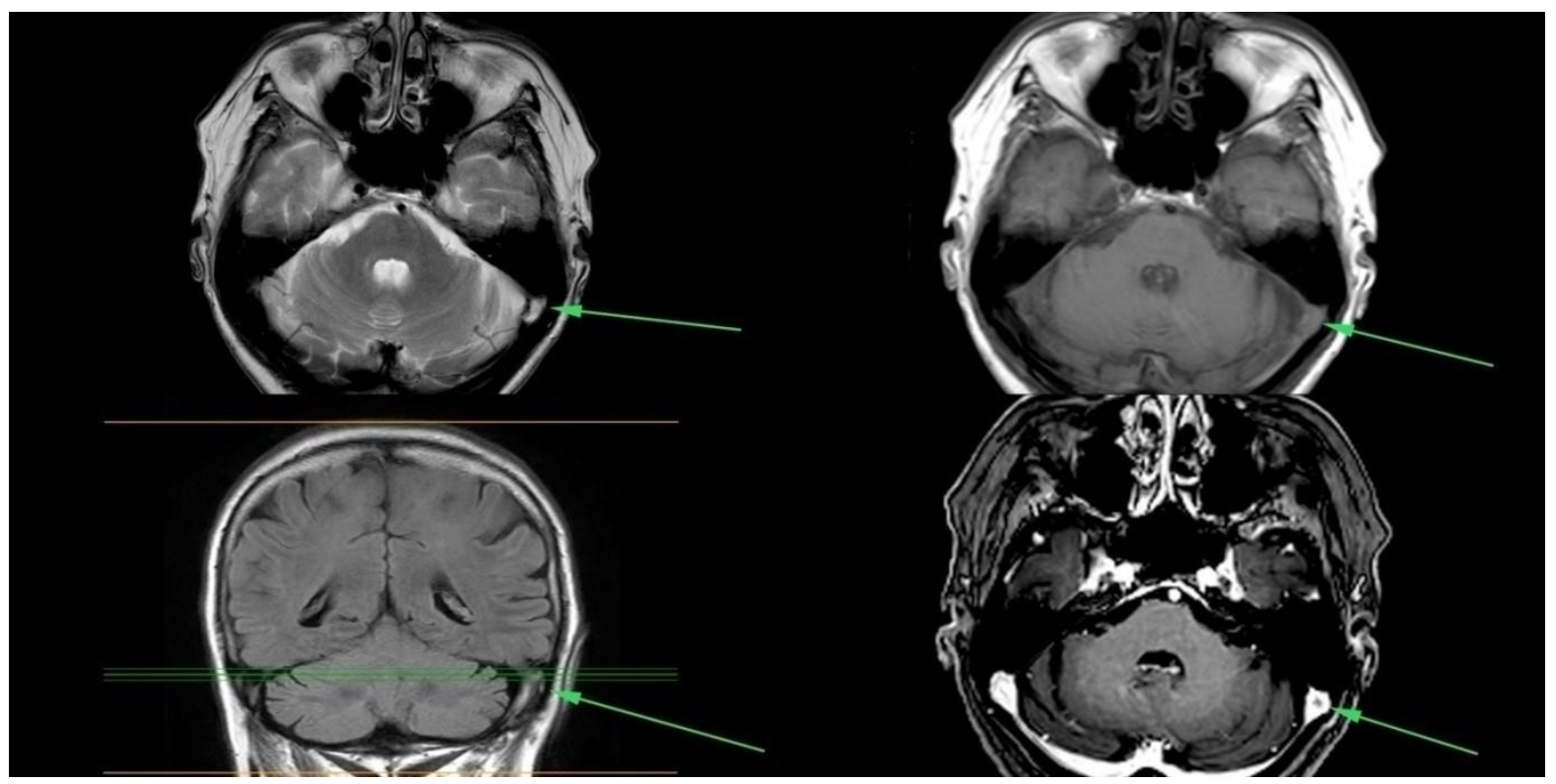

Figure 1. Brain MR and venography view; thrombosis in the left sigmoid sinus (arrow).

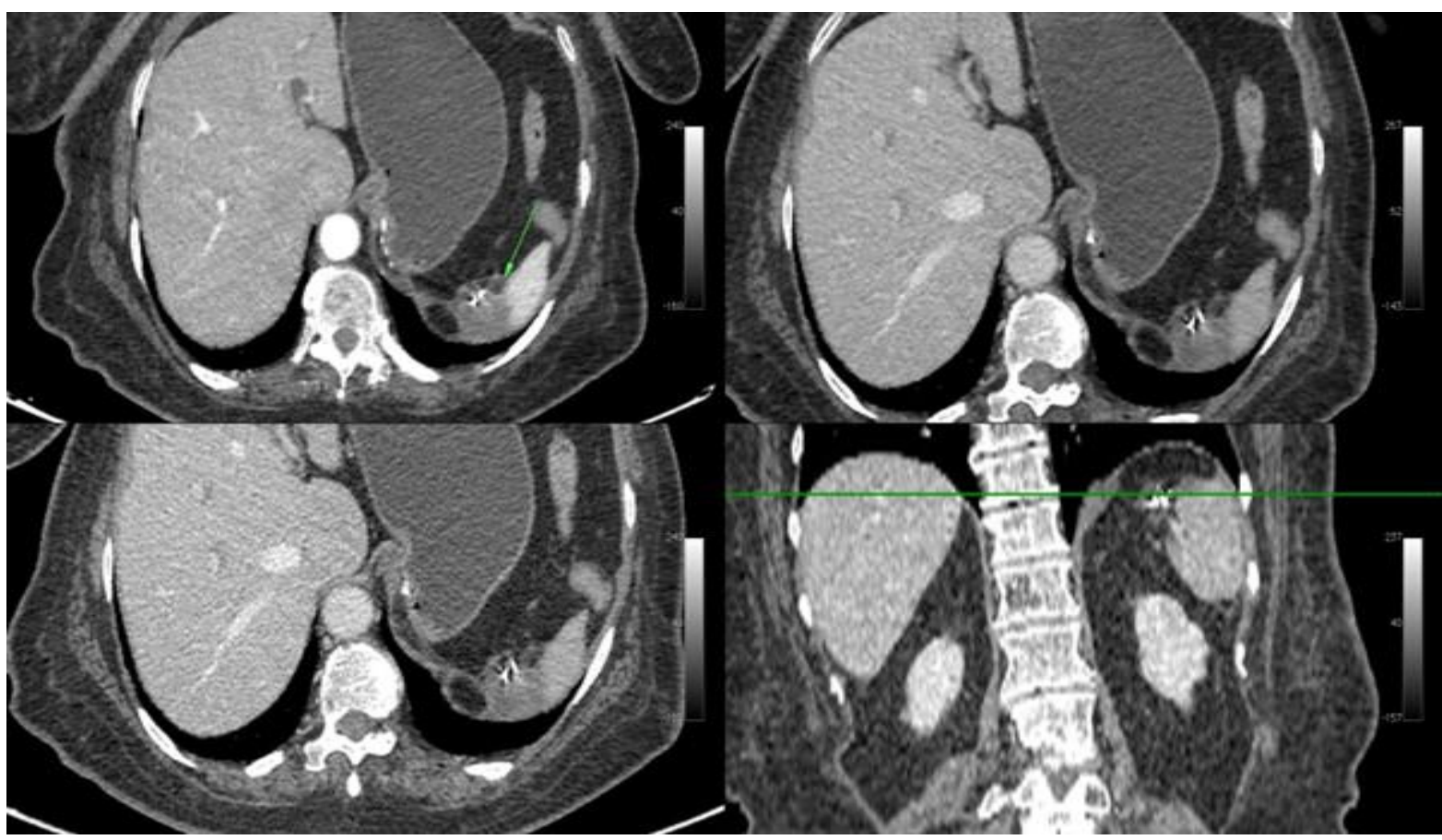

Figure 2. CT image of the abdomen. A wedge-shaped hypodense lesion in the spleen is compatible with splenic infarction (arrow).

patients, and hematological side effects such as anemia and leukopenia in 13 patients [7]. Theodotou et al. reported a case of spontaneous subdural hematoma associated with platelet dysfunction in a patient with GIST who was on imatinib therapy [8]. GIS hemorrhage was reported two weeks after imatinib therapy in a patient with duodenal GIST in whom hemorrhage could be controlled by surgical intervention [9].

However, thrombotic complications are extremely rare in patients with GIST receiving imatinib therapy. Therefore, a limited number of 
cases have been reported in the literature. In one of these studies, Chiang et al. reported spontaneous right axillosubclavian vein thrombosis in a patient with GIST receiving imatinib therapy who underwent percutaneous thrombectomy due to unresponsiveness to systemic anticoagulant therapy [10]. In another study, a fatal pulmonary thromboembolic event has been reported due to compression of the iliofemoral veins by necrotic metastatic tumor in a patient with metastatic GIST who was on imatinib therapy [11]. The present patient developed a series of thrombotic events after imatinib therapy. Unlike previously reported cases, however, our patient did not have any biochemical evidence or radiological signs of vascular compression indicating tumor necrosis. Although it is generally accepted that there is a tendency to thrombosis in cancer patients, some of which are partially attributed to paraneoplastic syndrome, there is no relationship between GIST and thrombotic paraneoplastic syndrome [12]. The absence of a history of thrombosis and genetic predisposition (protein $\mathrm{C}$, protein $\mathrm{S}$, antithrombin III normal, Factor $\mathrm{V}$ Leiden, methylenetetrahydrofolate reductase mutations, etc.) and conditions such as infective endocarditis, atrial fibrillation, hematological disorders (polycythemia vera, paroxysmal nocturnal hemoglobinuria, thrombotic thrombocytopenic purpura, etc.), autoimmune disorders, rheumatic diseases (SLE, Behçet, etc.) or predisposing infectious diseases supports the idea that imatinib therapy is the culprit for sigmoid sinus thrombosis and subsequent splenic infarction. In our case, imatinib is likely to have blocked PDGFR and caused ovarian expression of PDGF, thus resulting in thrombosis. However, the mechanism causing thrombosis in patients with GIST who are on imatinib therapy is still unknown, and further studies might be helpful in explaining this situation.

\section{CONCLUSIONS}

In conclusion, the current report presents the first case of sigmoid sinus thrombosis and subsequent splenic infarction, which complicate the treatment process with an unknown mechanism in patients with GIST receiving imatinib therapy. Although imatinib is considered to have fewer side effects than cytotoxic chemotherapeutics, it may be associated with thrombotic events such as sigmoid sinus thrombosis and splenic infarction. Therefore, thrombotic events should be kept in mind as a rare cause of headache and abdominal pain in patients with GIST receiving imatinib therapy, and appropriate clinical evaluation should be made accordingly.

\section{ACKNOWLEDGEMENT}

We acknowledge the patient and his brother for providing consent.

\section{CONFLICT OF INTERESTS}

The authors declare that they have no competing interests.

\section{REFERENCES}

1. Wang, S.Y., et al., Prospective Evaluation of Neoadjuvant Imatinib Use in Locally Advanced Gastrointestinal Stromal Tumors: Emphasis on the Optimal Duration of Neoadjuvant Imatinib Use, Safety, and Oncological Outcome. Cancers (Basel), 2019. 11(3). DOI: 10.3390/cancers11030424

2. Miettinen, M. and J. Lasota, Gastrointestinal stromal tumors: review on morphology, molecular pathology, prognosis, and differential diagnosis. Arch Pathol Lab Med, 2006. 130(10): p. 1466-78. DOI: $10.1043 / 1543-$ 2165(2006)130[1466:GSTROM]2.0.CO;2

3. Fu, Y., et al., Adverse reactions of sorafenib, sunitinib, and imatinib in treating digestive system tumors. Thorac Cancer, 2018. 9(5): p. 542-547. DOI: $10.1111 / 1759-7714.12608$

4. O'Brien, S.G., et al., Imatinib compared with interferon and low-dose cytarabine for newly diagnosed chronic-phase chronic myeloid leukemia. N Engl J Med, 2003. 348(11): p. 9941004. DOI: $10.1056 /$ NEJMoa022457

5. El Jurdi, N., et al., Perforation of the Colon During Imatinib Mesylate (Gleevec) Treatment in a Patient with Chronic Myeloid Leukemia (CML). Cureus, 2016. 8(6): p. e660. DOI: 10.7759/cureus.660

6. Reichardt, P., et al., Molecular response of gastrointestinal stromal tumour after treatment with tyrosine kinase inhibitor imatinib mesylate. J Clin Pathol, 2004. 57(2): p. 215-7. DOI: $10.1136 /$ jcp.2004.11379

7. Schlemmer, M., et al., Activity and side effects of imatinib in patients with gastrointestinal stromal tumors: data from a German multicenter trial. Eur J Med Res, 2011. 16(5): p. 206-12. DOI: $10.1186 / 2047-783 x-16-5-206$

8. Theodotou, C.B., et al., Subdural hematoma in a patient taking imatinib for GIST: a case report and discussion of risk with other chemotherapeutics. Anticancer Drugs, 2016. 27(3): p. 259-63.

DOI: $10.1097 / C A D .0000000000000325$ 
9. Hecker, A., et al., Dramatic regression and bleeding of a duodenal GIST during preoperative imatinib therapy: case report and review. World J Surg Oncol, 2010. 8: p. 47. DOI: 10.1186/1477-7819-8-47

10.Chiang, M.H., et al., Right axillosubclavian vein thrombosis resolved by percutaneous rheolytic thrombectomy in a patient with a recurrent gastrointestinal stromal tumour undergoing imatinib mesylate treatment. Phlebology, 2012. 27(7): p. 378-80. DOI: 10.1258/phleb.2011.011060

11.Melichar, B., et al., Fatal venous thrombembolism complicating imatinib therapy in a patient with metastatic gastrointestinal stromal tumor. J Exp Clin Cancer Res, 2006. 25(4): p. 607-10. PMID: 17310853

12.Tsikrikas, S., et al., Unusual combination of paraneoplastic manifestations in a patient with metastatic gastrointestinal stromal tumor (GIST). Scand J Gastroenterol, 2008. 43(8): p. 1012-5. DOI: 10.1080/00365520801886090 